TITLE:

\title{
Molecular dynamics and Monte- Carlo simulation of sputtering and mixing by ion irradiation
}

$\operatorname{AUTHOR}(S)$ :

Aoki, T; Chiba, S; Matsuo, J; Yamada, I; Biersack, JP

\section{CITATION:}

Aoki, T ...[et al]. Molecular dynamics and Monte-Carlo simulation of sputtering and mixing by ion irradiation. Nuclear Instruments and Methods in Physics Research Section B: Beam Interactions with Materials and Atoms 2001, 180(1-4): 312-316

\section{ISSUE DATE:}

2001-06

URL:

http://hdl.handle.net/2433/8947

\section{RIGHT:}

This is not the published version. Please cite only the published version.; この論文は出版社版でありません。引用の際には出版社版を ご確認ご利用ください。 


\section{Molecular Dynamics and Monte-Carlo Simulation of Sputtering and Mixing by Ion Irradiation}

T. Aoki ${ }^{1)}$, S. Chiba ${ }^{1)}$, J. Matsuo ${ }^{1)}$, I. Yamada ${ }^{\left.1)^{*}\right)}$ and J. P. Biersack ${ }^{2)}$

1) Ion Beam Engineering Experimental Laboratory, Kyoto University, Sakyo, Kyoto, 606-8501, Japan.

2) Hahn-Meitner Institute, Glienicker Strasse 100, D-14109, Berlin, Germany

e-mail of corresponding author: t-aoki@kuee.kyoto-u.ac.jp

*) Present Address: Laboratory of Advanced Science and Technology for Industry, Himeji Institute of Technology, CAST, Ako, Hyogo, 678-1205, Japan

\section{$\underline{\text { Abstract }}$}

Molecular dynamics (MD) and Monte-Carlo (MC) simulations of low-energy $(<500 \mathrm{eV})$ Ar ion irradiation on Si substrates were performed in order to investigate the mixing and sputtering effects. Both MD and MC simulation show similar results in sputtering yield, depth profile of projectile and mixing of substrate. For these incident energies, the depth of the mixed region is determined by the implant range of incident ions. For example, when the incident energy is $500 \mathrm{eV}$, the Ar ions reach a depth of $40 \AA$ so that the Si atoms that reside shallower than $40 \AA$ are fully mixed at an ion dose of about $5.0 \times 10^{16}$ atoms $/ \mathrm{cm}^{2}$. The resolution of secondary ion mass spectrometry (SIMS) was also studied. It was found that the resolution of SIMS depends on the depth of mixing, which depends in turn on the implant range of the probe ions. This is because the mixing of substrate atoms occurs more frequently than sputtering, so that the information about the depth profile in the mixing region is disturbed.

PACS $=79.20 . \mathrm{Rf}, 71.15 \mathrm{Pd}$

Keywords =Molecular dynamics simulation, Monte-Carlo simulation, SIMS resolution, ion mixing, sputtering

\section{$\underline{\text { Introduction }}$}

Secondary ion mass spectrometry (SIMS) is a useful technique to measure the depth profile of components in target materials [1]. Improving the resolution of SIMS analysis is required more and more recently because of the rapidly shrinking design rules in LSI fabrication. It is considered that the resolution of SIMS depends on the incident energy. In the collisional process of the probe atom, the incident atom induces a large number of collisions, so that the original distribution of substrate atoms is disturbed. With increasing incident energy, the disturbance of the original coordinates in the target becomes more frequent, and this causes a decrease in SIMS resolution. To perform high-resolution SIMS, it is necessary to use low-energy probe ions. However, as the incident energy decreases, it becomes difficult to obtain a sputtering yield which is sufficiently high for measurements. Therefore, the proper energy of the probe atom should be selected according to the required resolution. In this paper, the collisional processes of Ar atoms onto $\mathrm{Si}(001)$ substrate are simulated with both molecular dynamics (MD) and Monte-Carlo (MC) methods. The sputtering yield and the disturbance of delta-doped layer are compared. From these results, the dependence of SIMS resolution on the incident energy of probe ions will be discussed.

$\underline{\text { Simulation details }}$

In order to examine the mixing effects by ion beam irradiation, both molecular 
dynamics (MD) and Monte-Carlo (MC) simulations of Ar ions impacting on Si substrates were performed. For MD simulation, the Stillinger-Weber model potential [2] was applied to the interaction between Si atoms and the Ziegler-Biersak-Littmark (ZBL) model potential [3] was applied to Si and Ar atoms. The ZBL model was also used in the TRIM calculation. The cut-off radius of $\mathrm{Si}-\mathrm{Ar}$ interaction was set to $3.1 \AA$, which corresponds to the mean bond length of $\mathrm{Ar}-\mathrm{Ar}$ (in f.c.c. structure) and $\mathrm{Si}-\mathrm{Si}$ (in diamond). A $\mathrm{Si}(001)$ substrate, which consists of $2048 \mathrm{Si}$ atoms and has the dimensions of $20 \AA \times 20 \AA \times 90 \AA$, was prepared as the target material. In the simulation, $500 \mathrm{Ar}$ atoms were radiated on the substrate sequentially every $770 \mathrm{fs}$ with the impact point selected randomly on each impact. After irradiation with $500 \mathrm{Ar}$ atoms, the corresponding dose of Ar ions was calculated to be $1.25 \times 10^{16}$ atoms $/ \mathrm{cm}^{2}$. The substrate temperature was kept at $300^{\circ} \mathrm{C}$ by applying the Langevin dynamics method to the half bottom part of the substrate.

The MC simulations were also performed using the TRIM Dynamics (T-DYN) code $[4,5]$, which is based on TC (TRIM-CASCADE) [6] and was improved to be able to follow all recoil atoms, primary knock-on atoms and secondary knock-on atoms in the same way as it would follow the incident ions. The target substrate has an area of $1 \times 10^{5} \AA^{2}$ and a depth of $250 \AA$. The parameters describing substrate property are set to: $E_{b}$ (binding energy $)=2 \mathrm{eV}, E_{s b}$ (surface binding energy) $=3 \mathrm{eV}$ and $E_{d}$ (displacement energy) $=3 \mathrm{eV}$.

In both cases of MD and T-DYN simulation, Ar ions were radiated at normal incidence to the surface with energies of 100,200 and $500 \mathrm{eV}$. Si marker layers with thickness $2.5 \AA$, which corresponds to two mono-layers of $\mathrm{Si}(001)$, were positioned at depths of $0 \AA, 10 \AA, 20 \AA$ and $40 \AA$. The evolution of the mean depth and the deviation of each marker layer were examined in order to discuss the mixing effect by ion irradiation. The sputtered depth and the number of sputtered marker atoms provide the SIMS profile. From these SIMS profiles, the energy dependence of the SIMS resolution could be discussed.

\section{Results and discussion}

Fig. 1 shows the energy dependence of the sputtering yield of Ar ions to $\operatorname{Si}(001)$ substrate calculated by both MD and T-DYN. For incident energies below $1 \mathrm{keV}$, the sputtering yield rapidly decreases as the incident energy decreases. Fig. 2 shows the distribution of incident Ar ions by MD (Fig. 2a) and T-DYN (Fig. 2b). The mean implant depth and the deviation in each simulation are given in parentheses in the legends of Figs. $2 \mathrm{a}$ and 2 b. From Fig. 1 and Fig. 2, both T-DYN and MD show similar sputtering yields and implant profiles in the incident energy range of $100 \mathrm{eV}$ to $500 \mathrm{eV}$. These results indicate that the substrate property parameters for T-DYN are confirmed for these low energy ion impacts.

The evolution of the Si marker layer was investigated using the MD and MC methods. Fig. 3 shows snapshots of mixing by Ar ion with $500 \mathrm{eV}$ calculated by MD simulation. The atoms in the marker layer at $0 \AA, 20 \AA$ and $40 \AA$ are indicated as large circles and other substrate $\mathrm{Si}$ atoms are represented by small dots. On the other hand, the results by T-DYN calculations at $500 \mathrm{eV} \mathrm{Ar}$ ion irradiation with an atomic dose of $1.0 \times 10^{15}$ atoms $/ \mathrm{cm}^{2}$ (Fig. 4a) and $1.0 \times 10^{16}$ atoms $/ \mathrm{cm}^{2}$ (Fig. $4 \mathrm{~b}$ ) are shown in Fig. 4. It is shown in both MD and T-DYN calculation that, as the ion dose increases, the surface marker at $0 \AA$ expands to a deeper region in the substrate, whereas the layer at $20 \AA$ expands isotropically, and the deeper layer at $40 \AA$ moves towards the surface to a 
shallower region. At ion doses above $1.0 \times 10^{16}$ atoms $/ \mathrm{cm}^{2}$, these three makers show a similar profile in shape and the distribution of marker atoms is close to that of incident $\mathrm{Ar}$ atoms shown in Fig. 2.

Fig. 5 shows the dose dependence of the mean depth of each marker layer calculated by both MD and MC. The thick lines indicate the results by T-DYN calculation and symbols and error-bars are those obtained by MD calculation. The upper figures show the results with the dose ranging from 0 to $1.4 \times 10^{16}$ atoms $/ \mathrm{cm}^{2}$, and the lower ones show doses ranging to $1.0 \times 10^{17}$ atoms $/ \mathrm{cm}^{2}$. The transition of the surface level is calculated from the sputtering yield and is shown as thin lines. Both MD and T-DYN results show similar profiles for all marker layers. In the case of the irradiation with 100 and $200 \mathrm{eV}$, the marker layer at $40 \AA$ does not extend, which is different from the case of $500 \mathrm{eV}$. As the ion irradiation proceeds, the mean depth of each marker layer converges to the mean implant depth of the incident Ar atoms. Fig. 2 and 5 indicates close correlations between the implant depth and the mixing region. In the case of $200 \mathrm{eV}$ Ar ion irradiation, for example, the marker at $40 \AA$ moves rapidly towards the surface when the atomic dose is above $3.0 \times 10^{16}$ atoms $/ \mathrm{cm}^{2}$. This is because, as the sputtering proceeds, the depth of the marker at $40 \AA$ becomes more and more shallow. The sputtered depth at $3.0 \times 10^{16}$ atoms $/ \mathrm{cm}^{2}$ is calculated to be $8.2 \AA$, and thus the thickness of the mixing region could be estimated to be about $30 \AA(\approx 40 \AA-8.2 \AA)$. This thickness corresponds to the implant range of $\mathrm{Ar}$ ions with $200 \mathrm{eV}$, which in turn is calculated to be $28.1 \AA$ by the formula $(15.5 \AA$ (mean depth) $+2 \times 6.3 \AA$ (the deviation)). These results suggest that, with these low-energy ion implantations, the mixing of the implant region occurs faster than surface sputtering.

From the sputtering yield and the number of sputtered marker atoms, T-DYN simulations provide the SIMS response functions for the delta-doped maker layer. Fig. 6 shows the SIMS response functions by Ar ions accelerated with 100, 200 and 500eV. In all figures the dashed and dotted lines indicate the responses from the markers at $20 \AA$ and $40 \AA$, respectively. The solid-thick line is the sum of the two response lines in each figure, and is expected to represent a SIMS profile in real experiments. In any case, the signal from the marker at $20 \AA$ is detected at a lower dose than that expected from the sputtering yield, because of mixing of the surface region. In the case of $500 \mathrm{eV}$, the signal from $40 \AA$ is also detected at the sputter depth of $3 \AA$. This result means that both marker layers are mixed fully, as shown in Figs. 3, 4 and 5. As the incidence energy decreases, the penetration depth and the thickness of the mixed region are reduced. In the cases of 100 and $200 \mathrm{eV}$ of incident energy, the total signal shows two peaks, which can be divided into 2 different signals. Comparing the profiles at these incident energies, the signal from $20 \AA$ starts to increase at the sputtered depth of $0 \AA$ in both cases, whereas the signal from $40 \AA$ is detected at $14 \AA$ for $100 \mathrm{eV}$ and at $8 \AA$ for $200 \mathrm{eV}$. This result indicates that the thickness of the mixing layer is $26 \AA$ for $100 \mathrm{eV}$ and $32 \AA$ for $200 \mathrm{eV}$, which agrees with the results shown in Fig. 5. It is assumed that the resolution of SIMS measurement equals the thickness of mixing depth and also the implant range of probe ions.

\section{Conclusions}

The evolutions of marker layers in a Si substrate by sequential Ar ion radiations were investigated using the MD and MC methods. Both types of simulation show results consistent with each other in regard with sputtering yield, implant profile and mixing phenomena. At an incident energy of $500 \mathrm{eV}$, the surface region shallower than $40 \AA$ is 
mixed, so that the marker layer at $20 \AA$ extends itself isotropically, whereas the marker at $40 \AA$ goes up to a shallower region. The SIMS resolution depends on the depth of the mixed region and therefore depends on the incident energy of probe ion. From the T-DYN calculation, it was found that the energy of $200 \mathrm{eV}$, at which the Ar probe ion gives about $30 \AA$ of implant and mixing range, is the highest energy to resolve the marker separated with $20 \AA$ by SIMS analysis. As the incident energy decreases, the implant and mixing ranges are reduced, and the resolution is expected to improve further.

\section{References}

[1] Z. X. Jiang and P. F. A. Alkemade, J. Vac. Sci. Technol., B 16 (1998) 1971

[2] F. H. Stillinger and T. A. Weber, Phys. Rev. B, 31 (1985) 5262

[3] J. F. Ziegler, J. P. Biersack and U. Littmark, "The stopping and range of ions in solids", Pergamon Press, New York, 1985, p.321.

[4] J. P. Biersack, S. Berg, C. Nender, Nucl. Instr. and Meth. B, 59/60 (1991) 21

[5] J. P. Biersack, Nucl. Instr. and Meth. B, 133 (1999) 398

[6] J. P. Biersack and W. Eckstein, Appl. Phys. A, 34 (1984) 73 


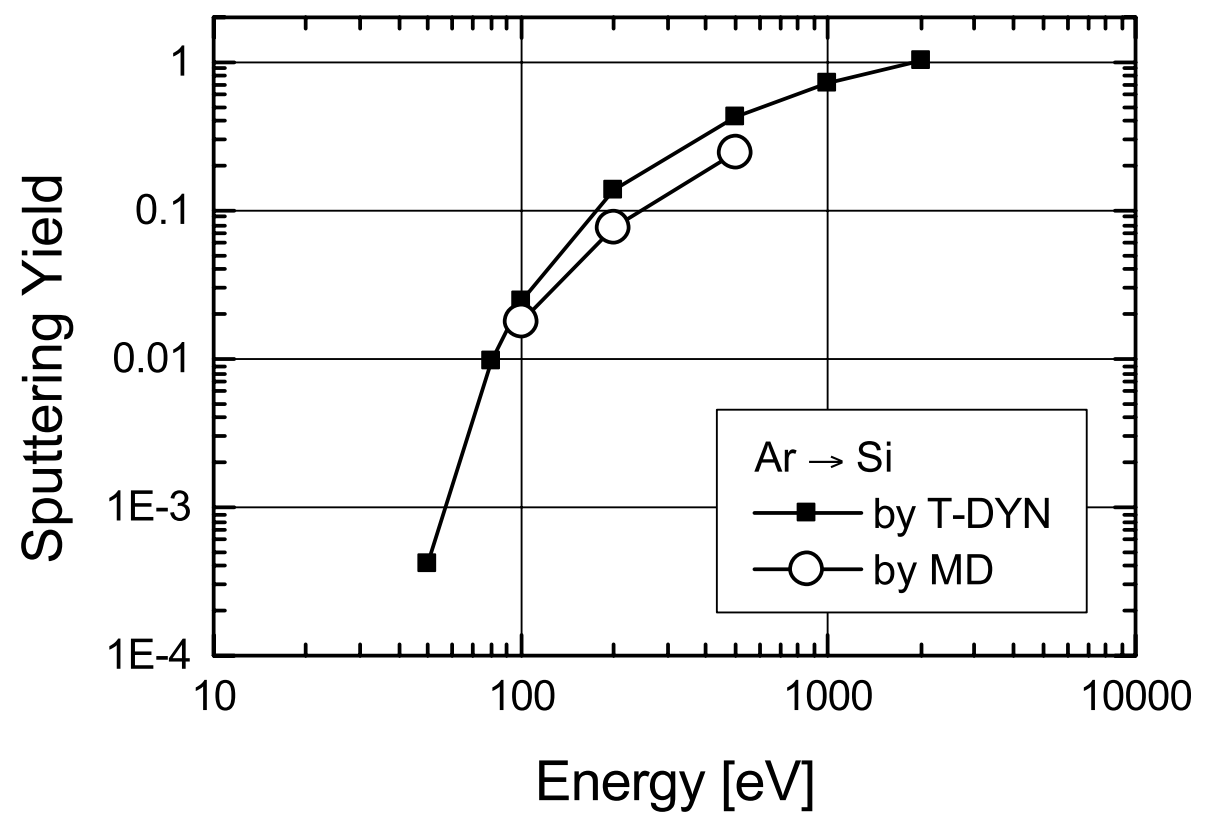

Fig. 1: Incident energy dependence of Si sputtering yield calculated by MD and T-DYN. 

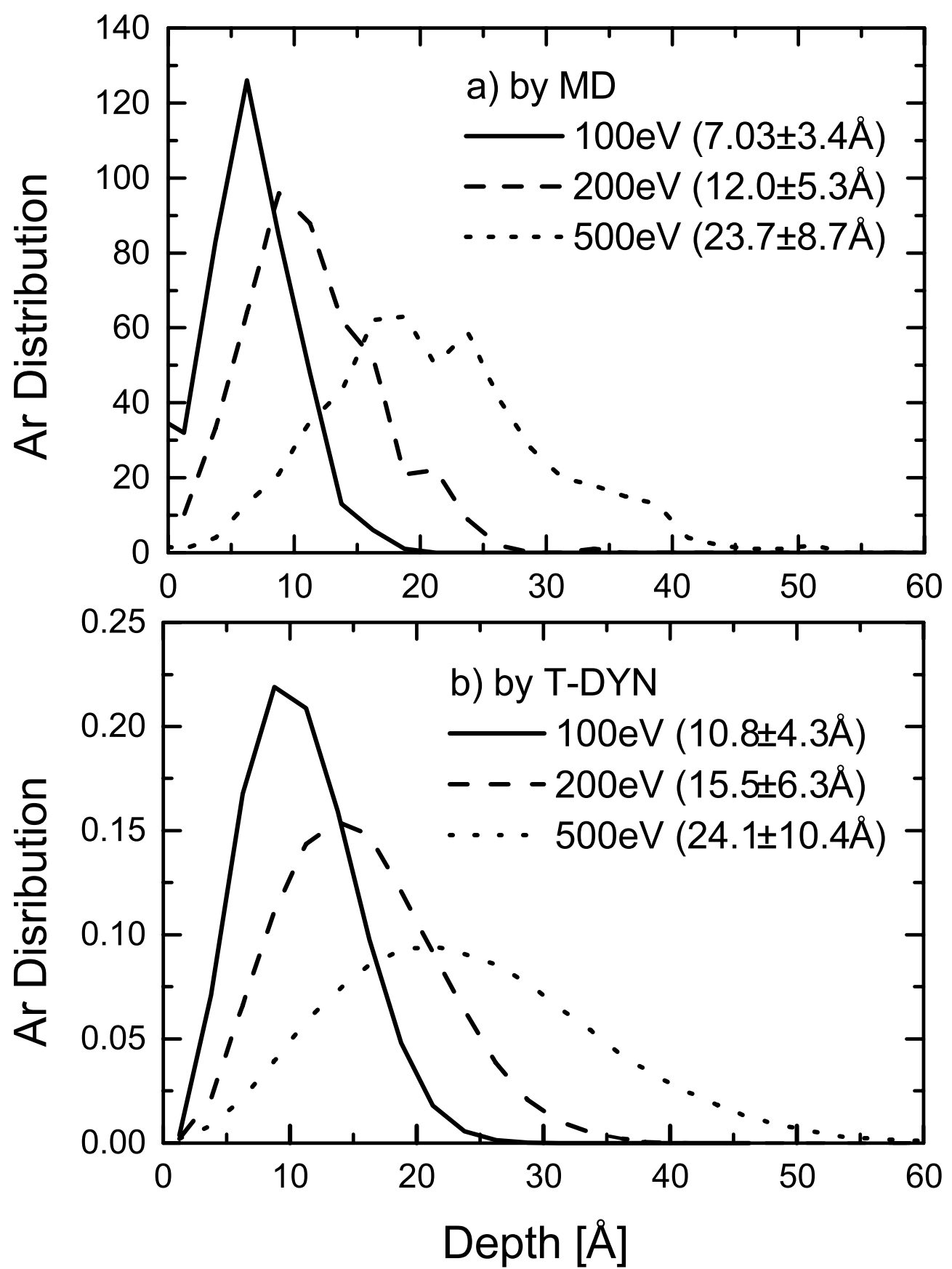

Fig. 2: Depth profile of implanted Ar ions calculated by MD (upper, a) and T-DYN (lower, b) 




Fig. 3: Snapshots of the mixing of Si substrate by Ar irradiation with $500 \mathrm{eV} /$ atom, calculated by $\mathrm{MD}$. Large circles represent the marker $\mathrm{Si}$ atoms, which are initially positioned at $0 \AA, 20 \AA$ and $40 \AA$



Fig. 4: Distribution of marker atoms calculated by T-DYN. The incident energy is $500 \mathrm{eV}$ and the atomic dose is (a) $1.0 \times 10^{15} / \mathrm{cm}^{2}$ and (b) $1.0 \times 10^{16} / \mathrm{cm}^{2}$. 

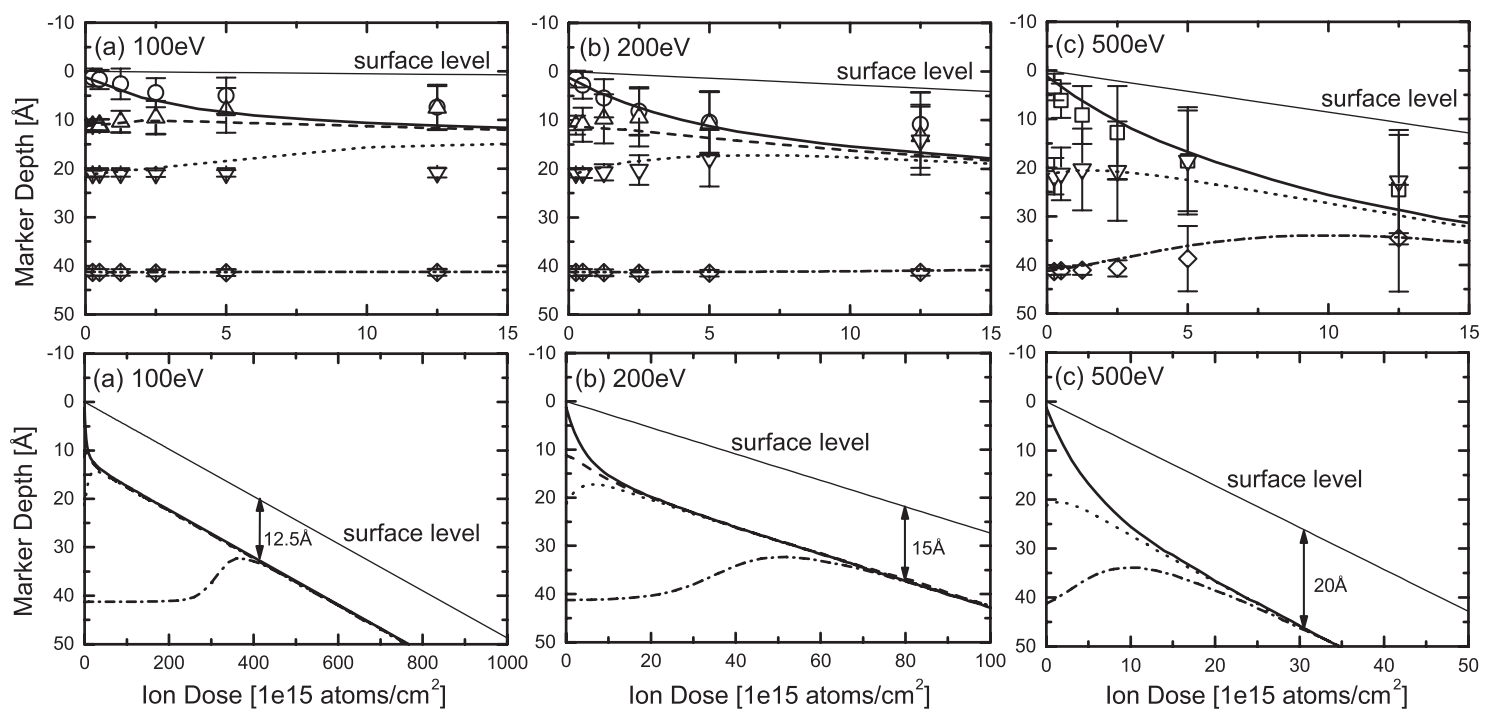

Fig. 5: Ion dose dependence of the mean depth of marker atoms with sequential Ar impacts with (a) $100 \mathrm{eV}$, (b) $200 \mathrm{eV}$ and (c) $500 \mathrm{eV}$. Lines and symbols represent the results by T-DYN and MD, respectively. The dose dependence of the sputtered depth is shown with thin line.
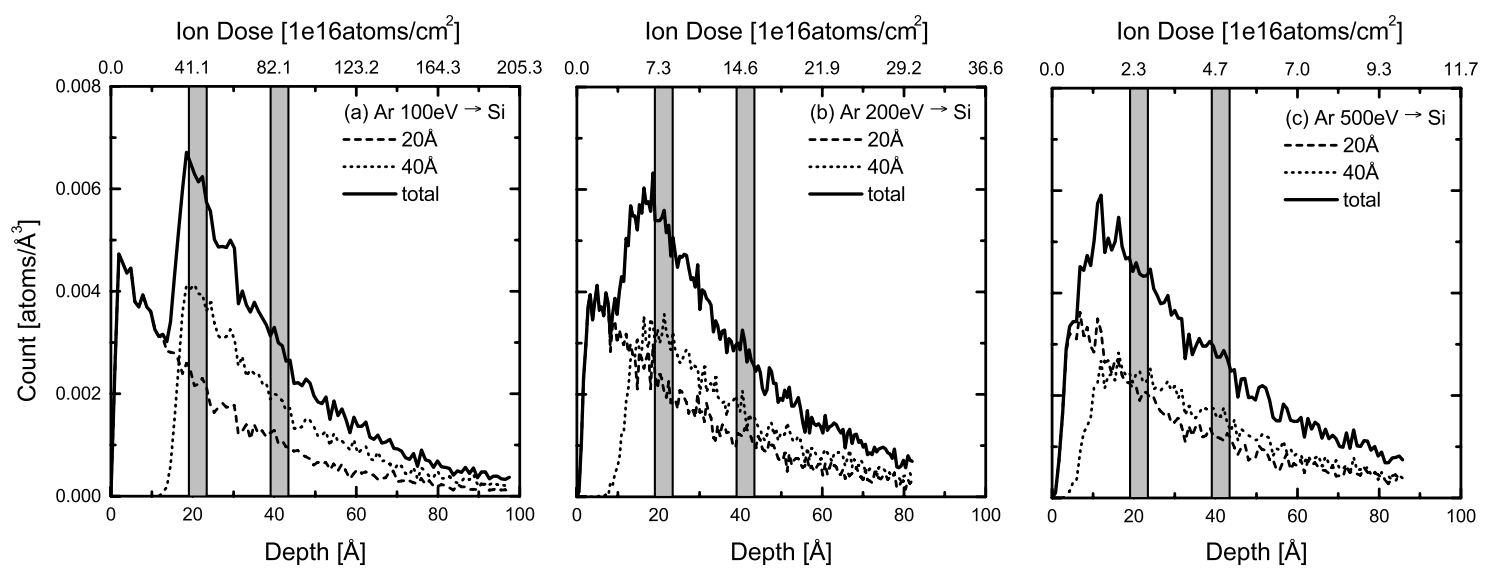

Fig. 6: SIMS response profile of two marker layers calculated by T-DYN. The incident energy is (a) $100 \mathrm{eV}$, (b) $200 \mathrm{eV}$ and (c) $500 \mathrm{eV}$. 